\title{
A Cross-National Study of Dementia Stigma Among the General Public in Israel and Australia
}

\author{
Perla Werner ${ }^{\mathrm{a}, *}$ and Sarang Kim ${ }^{\mathrm{b}}$ \\ ${ }^{a}$ Department of Community Mental Health, University of Haifa, Haifa, Israel \\ ${ }^{\mathrm{b}}$ Wicking Dementia Research \& Education Centre, University of Tasmania, Hobart, Australia
}

Handling Associate Editor: Sid O’Bryant

Accepted 5 June 2021

Pre-press 9 July 2021

\begin{abstract}
.
Background: Despite the increasing amount of research on dementia stigma, there is a dearth of cross-national studies conducted on this subject. This is surprising since the experience of stigma is closely associated to socio-cultural aspects.

Objective: The present study intended to expand knowledge about the impact of culture on dementia stigma by comparing the level and correlates of stigmatic beliefs about dementia among the general public in Israel and Australia.

Methods: A cross-sectional study using an online survey was conducted with two age-matched samples: 447 adults in Israel and 290 adults in Australia.

Results: Overall, dementia stigma was moderate in both countries. However, the level of dementia stigma was significantly higher in Australia than in Israel. Lower levels of subjective knowledge and higher levels of ageism were associated with increased levels of stigmatic beliefs in both countries. Gender was a significant correlate of dementia stigma, with male participants reporting higher levels of public stigma than women, although this gender difference was mainly driven by the Australian sample.

Conclusion: Our findings indicate that providing knowledge and decreasing ageist attitudes should be key considerations in dementia awareness and stigma reduction campaigns despite the cultural context. In addition, developing gender-specific messages should be considered as a way of improving the effects of such campaigns.
\end{abstract}

Keywords: Ageism, cross-national study, dementia, dementia knowledge, public stigma

\section{INTRODUCTION}

Dementia-related stigma has been given increased policy and research attention over the past decade as a result of rapid growth in the aging population [1] and a rise in the number of people living with dementia. Dementia-related public stigma has been defined as the cognitive, emotional, and discriminatory attributions endorsed by the general public

\footnotetext{
*Correspondence to: Perla Werner, PhD, Department of Community Mental Health, University of Haifa, Mt. Carmel, Haifa, Israel. Tel.: +972 54 3933066; E-mails: werner@ research.haifa. ac.il; pwerner@univ.haifa.ac.il
}

toward people with dementia. It was found to be associated with deleterious consequences such as decreased help-seeking, and discrimination in the allocation of resources in the health, welfare, and legal systems which might lead to improper care to people with dementia [2-4]. Consequently, National Dementia Strategy programs worldwide have defined decreasing dementia stigmatic beliefs among the general public as a core goal [5]. A rising number of researchers from various countries have examined the level and correlates of these beliefs among laypersons in their societies $[2,3]$.

Overall, as concluded in a recent review [4], studies on dementia stigma among the general public 
have found the levels of stigmatic attitudes to be moderate [6-9]. Where stigma exists, people with dementia are perceived as unpredictable and difficult to communicate with [10] and as having negative aesthetics attributes [11-13]. In terms of emotional reactions, studies on dementia stigma among lay persons showed that people with dementia elicit feelings of shame and fear [7, 8, 14-16], but also positive feelings of pity and willingness to help $[12,17]$. Furthermore, the most common discriminatory behaviors reported by the general public included social distancing, discrimination in relation to employment and health insurance [11, 12], and the belief that persons with dementia should be institutionalized [18]. As for the factors associated with dementia stigma, lower education, belonging to a minority group, and familiarity with a person with dementia were consistently associated with lower levels of stigmatizing attitudes [7, 9, 18-23].

Despite this impressive amount of research on dementia stigma, there is a dearth of cross-national studies conducted on this subject [3]. This is surprising since comparative studies in the area of mental illness have demonstrated that stigmatic beliefs are shaped by cultural factors [24]. Moreover, perceptions of dementia are socially constructed and influenced by socio-cultural and political contexts [25]. Yet, to the best of our knowledge, only two studies have compared dementia stigma among the general public in different countries. One study was conducted among older adults, aged 65 and over, attending primary care services in the United Kingdom and the United States. Results indicated that participants from the United Kingdom reported higher dementia stigma scores than older adults from the United States [26]. Another study, conducted among college students in Greece and Israel, found higher levels of dementia stigma among Israeli students than among Greek students [13]. Interestingly, however, while a higher level of familiarity with dementia was associated with higher levels of stigma held by older people, dementia stigma was associated with lower levels of familiarity among college students. Given the paucity and inconsistent findings of these crossnational studies, we intended to expand knowledge about the impact of culture on dementia stigma by comparing the level and correlates of stigmatic beliefs about dementia among the general public in Israel and Australia.

Both Israel and Australia are multicultural societies, intricately composed of various groups of immigrants and indigenous populations. Demographically, compared to Australia, Israel is a younger country. Data from the World Bank [27] shows that $12 \%$ of the population in Israel is aged 65 and above, compared to $16 \%$ in Australia. Accordingly, in terms of the prevalence of dementia, there are 10 and 14 people per 1000 people living with dementia in Israel and Australia, respectively [28]. Since these numbers are expected to increase in future years, both countries have initiated national strategic programs to improve the quality of life and care provided to people living with dementia and their caregivers, to reduce the risk of dementia, and to increase awareness about the disease $[29,30]$. Culturally, Australia is highly individualistic, whereas Israel is only moderately so, according to Hofstede's ranking of countries [31]. Since the level of individualism in a culture has been found to be associated with lower levels of stigma in the area of mental illness [32], we hypothesized that dementia stigma will be higher in Israel than in Australia.

Furthermore, this study examined knowledge about dementia, ageism, and familiarity with dementia as factors contributing to dementia stigma. First, higher levels of knowledge about the disease have been reported to be associated with lower levels of stigmatic attitudes [33-35]. Second, the stigma associated with old age was found to reinforce dementia stigma, reflecting a phenomenon called "double stigma" [36]. Finally, while for many years having more familiarity with persons with a disease was hypothesized as being associated with decreased stigma [37], lately, the direction of this association is controversial for both mental illnesses [38], and for dementia stigma $[13,26]$. Based on this literature, we hypothesized that higher levels of dementia knowledge and lower levels of ageism, will be significantly associated with lower levels of dementia stigma. Based on the inconsistent findings about the relationship between familiarity and dementia stigma, no hypothesis was proposed for this association.

\section{METHODS}

\section{Design and procedure}

A cross-sectional, online survey was conducted with two age-matched samples (447 adults in Israel and 290 adults in Australia), using a similarly structured survey. Inclusion criteria included being at least 18 years old, and literate in the language of the questionnaire. There were no further inclusion or exclusion criteria. 
Israeli participants were recruited from a comprehensive internet panel through a major Israeli internet panel company (PValue), which maintains panels of potential participants for the entire Israeli population. All active and potentially eligible panelists according to the study requirements were identified by the company by a special matrix. Potential respondents were invited to participate in the online survey for a limited period (June 20 to June 24, 2018). Once quotas by gender, age, and region were reached for each parameter, the survey was closed. For the Australian sample, a similar approach was taken. A web address for the online survey was distributed to a nationally representative sample by the survey company, Qualtrics. Anyone who was 18 years or older who was registered to be in the Qualtrics participant pool was able to participate in this online survey. Quotas were set up to match the census distribution on age and gender. The data were collected from June 15 to June 19, 2018.

\section{Instruments}

\section{Stigma toward dementia}

Similar to previous studies [39], stigma toward dementia was examined using nine items assessing the cognitive, emotional, and behavioral aspects of public stigma. Example items included: "People with dementia should be put into a nursing home", "I am afraid of people with dementia", and "I will try to keep distance from people with dementia". All of the items were rated on a 9-point Likert-type scale, ranging from "strongly disagree" (1) to "strongly agree" (9). An overall index was calculated by averaging 6 out of the 9 items. This index provided the best internal reliability in both samples (Cronbach alpha $=0.64$ and 0.76 for Israel and Australia, respectively).

\section{Subjective knowledge about dementia}

Similar to Werner and colleagues [40], participants were asked to report how much they think they know about dementia on a 5-point Likert-type scale, ranging from "not much at all" (1) to "very much" (5).

\section{Ageism}

This was assessed by using the 18-item Hebrew version of the Fraboni Scale of Ageism [41] among the Israeli sample and the original 20-item scale [42] among the Australian sample. Twelve common items were found between the two versions of the questionnaires and used in the analyses in the current study. Example items included "I don't like it when old people try to make conversation with me", "Old people should find friends their own age", "Many old people are stingy and hoard their money and possessions". Participants rated each item on a 4-point Likert-type scale, ranging from "strongly disagree" (1) to "strongly agree" (4). Moderate internal reliability was found for these items in both countries (Cronbach's alphas $=0.68$ in the Israeli sample and 0.63 in the Australian sample). Thus, an overall index of the mean score of the ageism items was calculated, with high scores demonstrating higher levels of ageism.

\section{Familiarity with dementia}

Similar to Werner and colleagues [40], familiarity with dementia was assessed by asking participants whether or not they knew someone with Alzheimer's disease.

\section{Background information}

This included information about the participants' gender (male/female), age, and ethnicity, i.e., majority (Jewish for Israel and British and European for Australia) or minority (Arabs for Israel and all other ethnic groups for Australia).

\section{Statistical analyses}

A comparison of the characteristics of the Israeli and Australian samples, as well as of the main study variables, was conducted using independent samples $t$-tests and chi-square tests. To examine the associations between the study variables and dementia stigma in the total sample, a multiple linear regression was conducted. At the first step, background variables that were related with dementia stigma were entered as control variables (gender, ethnicity, and familiarity with dementia). At the second step, subjective knowledge of dementia, and ageism were entered. At the third step, the country variable was entered. Finally, at the fourth step, interactions between country and each of the independent variables were entered in a stepwise manner. All continuous variables were standardized.

\section{Ethical considerations}

The study's protocol was approved by the Ethics Committee of the University of Haifa and the Australian National University. 
Table 1

Descriptive statistics of socio-demographic and study variables

\begin{tabular}{|c|c|c|c|}
\hline & $\begin{array}{c}\text { Israeli sample } \\
\quad(n=447)\end{array}$ & $\begin{array}{c}\text { Australian sample } \\
\quad(n=290)\end{array}$ & Comparison \\
\hline \multicolumn{4}{|l|}{ Socio-demographic characteristics } \\
\hline Gender ( $\%$ Female $)$ & 50.1 & 50.2 & $\chi_{(1)}^{2}=0.01, p>0.05$ \\
\hline Age & $42.48(13.10)^{\mathrm{a}}$ & $43.67(14.21)^{b}$ & $\mathrm{t}_{(1,581)}=-1.15, p>0.05$ \\
\hline Ethnicity (\% Majority) & 77.9 & 83.9 & $\chi_{(1)}^{2}=4.04, p<0.05$ \\
\hline \multicolumn{4}{|l|}{ Study variables } \\
\hline $\begin{array}{c}\text { Familiarity with someone } \\
\text { with dementia (\% yes) }\end{array}$ & 52.1 & 36.6 & $\chi_{(1)}^{2}=16.84, p<0.001$ \\
\hline $\begin{array}{l}\text { Mean (SD) subjective knowledge } \\
\text { of dementia (range: } 1-5 \text { ) }\end{array}$ & $3.17(0.92)$ & $2.57(0.89)$ & $\mathrm{t}_{(1,716)}=8.75, p<0.001$ \\
\hline Mean $(\mathrm{SD})$ ageism $^{\mathrm{c}}$ & $2.05(0.38)$ & $1.85(0.42)$ & $\mathrm{t}_{(1,587)}=6.33, p<0.001$ \\
\hline Mean (SD) dementia stigma ${ }^{\mathrm{d}}$ & $2.44(1.14)$ & $3.04(1.26)$ & $\mathrm{t}_{(1,578)}=-6.42, p<0.001$ \\
\hline
\end{tabular}

a Range: $24-70 .{ }^{b}$ Range: $18-69 .{ }^{\mathrm{c}}$ Mean range: $1-4 .{ }^{\mathrm{d}}$ Mean range: $1-9$.

\section{RESULTS}

\section{Participants' socio-demographic characteristics}

As can be observed in Table 1, the sample included 447 participants from Israel and 290 participants from Australia. Among Israeli participants, 49.9\% were male and $50.1 \%$ female, and among Australians, $49.8 \%$ were male and $50.2 \%$ were female. The mean age in the Israeli sample was not significantly different than in the Australian sample. A larger proportion of the Australian sample reported themselves to be part of an ethnic majority $(83.9 \%)$ than the Israeli sample (77.9\%), indicating that the Israeli sample had more participants who defined themselves as belonging to ethnic minorities.

\section{Comparison between Israeli and Australian samples in study variables}

A comparison between the Israeli and Australian age-matched samples (Table 1) showed several differences between the countries in the study's main variables. First, regarding dementia stigma and based on bivariate analyses, Israeli participants reported statistically significant $(p<0.001)$ lower levels of stigma toward dementia in comparison with Australian participants in the overall index. Second, the Israeli sample reported having statistically significant $(p<0.001)$ greater perceived knowledge regarding dementia and higher levels of ageism $(p<0.001)$, compared to the Australian sample. Finally, a larger percentage of the Israeli sample reported being familiar with someone with dementia compared to the Australian sample $(p<0.001)$.

\section{Associations of dementia stigma with independent variables in the total sample}

Dementia stigma was higher among males $(M=$ $2.81, S D=1.27)$ than females $(M=2.56, S D=1.17)$ $\left(t_{(708)}=2.75, p=0.006\right)$, and among minority participants $(M=2.92, S D=1.38)$ than majority ones $(M=2.63, S D=1.18) \quad\left(t_{(188.37)}=2.20, \quad p=0.029\right)$. Further, it was higher among those not familiar with someone with dementia $(M=2.82, S D=1.29)$ than among those familiar with someone with dementia $(M=2.53, S D=1.13)\left(t_{(708.04)}=3.21, p=0.001\right)$. However, it was not significantly associated with participants' age $(r=-0.06, p=0.094)$.

Table 2

Multiple regression analysis for dementia stigma and its association with background and predictor variables in the total sample ${ }^{1}(n=693)$

\begin{tabular}{lcccc}
\hline Independent variables & Model 1 & Model 2 & Model 3 & Model 4 \\
\hline Gender (male) & $0.10^{* *}(0.02,0.17)$ & $0.03(-0.03,0.10)$ & $0.03(-0.03,0.09)$ & $0.03(-0.03,0.09)$ \\
Ethnicity (majority) & $-0.09^{*}(-0.16,-0.01)$ & $-0.06(-0.13,0.01)$ & $-0.06(-0.13,0.01)$ & $-0.06(-0.13,0.01)$ \\
Familiarity with dementia & $-0.11^{* *}(-0.18,-0.04)$ & $-0.03(-0.11,0.04)$ & $-0.01(-0.08,0.05)$ & $-0.01(-0.08,0.05)$ \\
Subjective knowledge & & $-0.20^{* * *}(-0.28,-0.13)$ & $-0.11^{* *}(-0.18,-0.04)$ & $-0.11^{* *}(-0.18,-0.04)$ \\
$\quad$ about dementia & & & & $0.39^{* * *}(0.32,0.46)$ \\
Ageism & & & $0.46^{* * *}(0.40,0.53)$ & $0.45^{* * *}(0.39,0.52)$ \\
Country (Israel) & & $-0.32^{* * *}(-0.39,-0.25)$ & $-0.32^{* * *}(-0.39,-0.25)$ \\
Country X gender & & & $-0.10^{* *}(-0.16,-0.03)$ \\
Cumulated $R^{2}$ & $\mathbf{0 . 0 3}^{* * *}$ & $\mathbf{0 . 2 0}^{* * *}$ & $\mathbf{0 . 2 9}^{* * *}$ & $\mathbf{0 . 3 0}^{* * *}$ \\
\hline
\end{tabular}

\footnotetext{
${ }^{1}$ Numbers in the table are standardized coefficients ${ }^{* * *} p<0.001^{* *} p<0.01 * p<0.05$.
} 
Table 2 displays the results for the multiple regression. As can be observed, while gender, ethnicity, and familiarity with dementia were statistically significant in the first step of the analysis, their effects disappeared in the second step. After controlling for gender, ethnicity, and familiarity with dementia, the variables entered in the second step (subjective knowledge about dementia and ageism) were significantly associated with dementia stigma and increased the explained variance in dementia stigma by $17 \%$. When the country of residence was included in the model (step 3), $R^{2}$ increased by $9 \%$ (from $20 \%$ to $29 \%$ ); the interaction effect added another $1 \%$ to the explained variance in step 4 . That is, lower levels of subjective knowledge about dementia, and increased ageism, were significantly associated with increased dementia stigma.

Only one interaction term was found significant: that between country and gender. As it involves two dichotomous variables, it was interpreted with an analysis of covariance and estimated marginal means. The analysis revealed that while Australian men had a higher dementia stigma mean $(M=3.35$, $S E=0.09)$ than Australian women $(M=2.98, S E=$ 0.09) $\left(F_{(1,685)}=8.51, p=0.004, \eta^{2}=0.012\right)$, the gender difference was not significant within the Israeli sample (Israeli men: $M=2.30, S E=0.07$, Israeli women: $M=2.42, S E=0.07, F_{(1,685)}=1.36$, $p=0.244, \eta^{2}=0.002$ ) (Fig. 1). That is, Australian men had the highest dementia stigma mean, which in part explains the higher stigma mean in the Australian sample compared to the Israeli sample. These results should be interpreted with caution due to the low effect sizes.

It should be noted that splitting the regression analysis between the two countries has revealed identical results. Significant factors in the Israeli

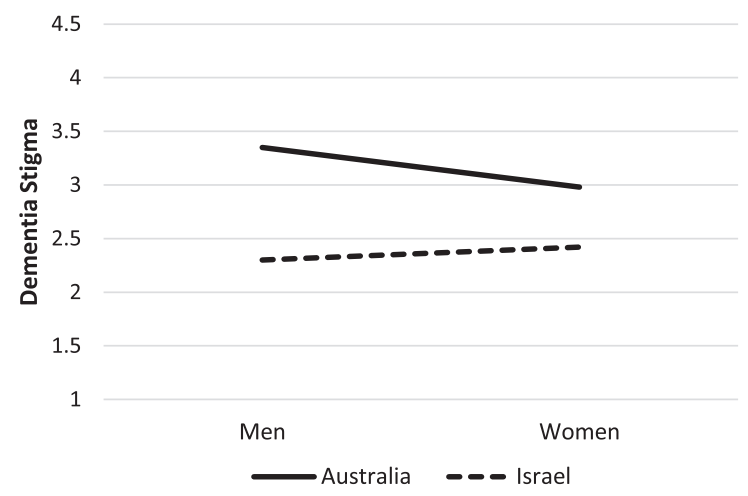

Fig. 1. Dementia stigma by country and gender. sub-sample were subjective knowledge about dementia $(\beta=-0.13, p=0.006)$, and ageism $(\beta=0.41, p<$ 0.001 ), while significant factors in the Australian sub-sample were subjective knowledge about dementia $(\beta=-0.13, \quad p=0.013)$, ageism $(\beta=0.49$, $p<0.001)$, and gender $(\beta=0.13, p=0.014)$.

\section{DISCUSSION}

Since the experience of stigma is closely associated to societal aspects, abundant research tries to understand the origins and process of mental illness stigma using cross-national designs [43]. In the area of dementia, however, there is significantly less research comparing stigmatic attributions toward persons with the disease across countries and cultures. The current study set out to expand this limited knowledge by examining dementia stigma and its correlates as reported by the general public in Israel and Australia.

Overall, levels of dementia stigma were moderate in both countries-a mean of 2.4 and 3.0 in Israel and Australia respectively, within scores ranging 1 through 9. This is in line with the World Alzheimer Report [45] showing that people in developed countries have relatively low levels of dementia stigma. Although consistent with previous studies conducted in various countries (see Werner and her colleagues [4]), it would be helpful to understand the roots of these findings. One possible explanation at the individual level are the low levels of responsibility and negative emotions consistently attributed to a person living with dementia [7, 12, 45].

Another explanation, however, might stem from the characteristics of the countries rather than from the stigmatic beliefs of their citizens. Both Israel and Australia are Western countries characterized by concern toward people with dementia. This concern is reflected in the implementation of strategic national plans on dementia [46], and by the availability of care frameworks for persons with the disease and their family members $[47,48]$.

Finally, we cannot rule out methodological explanations for these findings. First, the use of an overall index averaging cognitive, emotional, and behavioral aspects of stigma might have influenced the level of stigma found in both countries. Indeed, studies using individual items to assess stigmatic beliefs have shown higher levels of stigma in Israel [18], as well as in Australia [15]. Second, similar to other cross-national studies using self-report 
measures [49], response biases in general and social desirability in particular might have affected participants' responses.

Regarding our first hypothesis, contrary to our expectations, the level of dementia stigma was significantly higher in Australia than in Israel. This finding is identical to the one reported in our previous cross-national study, in which contrary to our hypothesis higher levels of stigma were found in the more individualistic country-Israel-than in the less individualistic one-Greece [13]. Several explanations might be provided for this finding. First, the ranking of both countries in the individualisticcollectivistic dimension was reported in 2008 and no data are available since. However, Israel is becoming a more individualistic and even capitalistic society [50]. Moreover, lately the individualism-collectivism division to explain differences among cultures is being criticized as assuming too much homogeneity and same-mindedness as characterizing different cultures [51, 52]. Thus, Israel and Australia, being two Western cultures, might be more similar in their cultural values than hypothesized, which might be reflected in the levels of public stigma.

Second, the statistically significant difference found in the level of stigma reported by the general public in both countries, might be a consequence of the characteristics of the samples rather than a reflection of stigmatic beliefs. The Australian sample being less familiar with people with dementia, and having lower subjective dementia knowledge, may lead to higher levels of dementia stigma among Australians. Indeed, our findings show that the level of stigma was affected by the interaction of gender and country, with Australian men reporting the higher level of stigma. Previous Australian research has also suggested that dementia-related stigma is specific to gender where being male was significantly associated with higher levels of dementia stigma [53].

In terms of the determinants of dementia stigma, lower levels of subjective knowledge and higher levels of ageism were, as hypothesized, associated with increased levels of stigmatic beliefs in both countries. These findings add to the existent literature in various aspects. Firstly, regarding knowledge, the findings demonstrate that stigma is associated not only with what people actually know (i.e., objective knowledge) [34], but also with what they think they know. This suggests that interventions aimed at decreasing stigmatic beliefs regarding dementia should not be limited to the provision of factual knowledge, but also to increasing participants' confidence in the knowledge they have on the matter. Secondly, regarding ageism, our findings support empirically the conceptually established association between ageism and dementia stigma $[3,36]$, underscoring the need to invest effort in changing the attitudes towards elderly persons in general, as an antecedent to stigmatic beliefs toward persons with dementia. Thirdly, in this study, familiarity with a person with dementia was not significantly associated with stigmatic beliefs. Indeed, as previously stated, findings about the association of this variable with mental illness stigma are inconsistent. Moreover, it has been recently suggested that this might be a U-shaped relationship rather than a linear one [38]. Future studies should examine empirically this relationship in the area of dementia stigma.

Finally, gender was a significant determinant of dementia stigma in our study, with male participants reporting higher levels of public stigma than women, although this gender difference was mainly driven by the Australian sample. This is an important finding as studies examining gender differences in public stigma toward mental illnesses have shown inconsistent results, with some studies reporting higher levels of stigma in males, others in females, and others no gender differences [54]. Our results might be the result of females' general tendency to higher levels of empathy [54], or to the fact that females assume traditionally the role of the main informal caregiver of persons with dementia [55].

Before discussing the implications of this study, we have to acknowledge several limitations. First, the use of a cross-sectional design limits our capability to draw causal conclusions. Second, we used structured validated measures; however, we still relied on selfreported data. Although this limitation might result in an increased social desirability bias, we believe the anonymity of the survey safeguarded the trustworthiness of the reports. Third, given the age of the samples, we cannot disregard the results being affected by their cognitive status or unconscious beliefs towards people with $\mathrm{AD}$, i.e., implicit bias. Fourth, future studies should examine relationships with other important socio-demographic variables such as education and income level. Finally, the use of qualitative methods could have helped deepen our understanding of the differences in stigmatic attributions about dementia between the two countries.

Despite these limitations, this study enhances the understanding of dementia stigma on several levels. First, conceptually, our findings provide support to previous quantitative studies showing, contrary to 
qualitative and anecdotal data, that dementia stigma is relatively low among the general public. While quantitative studies in the area of mental-health stigma show similar results [56], there is need to expand the efforts to understand these patterns, while examining the appropriateness of current conceptualizations of stigma in the area of dementia. Second, since health providers are not only part of the general public but also are affected by its attitudes and beliefs, expanding knowledge in this area might lead to an early diagnosis of the condition and to better care. Third, in regard to interventions for reducing dementia stigma among the general public, our findings indicate that providing knowledge, and decreasing ageist attitudes should be key considerations in dementia awareness and stigma reduction campaigns. Moreover, special attention should be paid to the gender of the target population, and consideration given to developing gender-specific messages as a way of improving the effects of the intervention. Finally, there is need to expand comparative, cross-national research among culturally and socially similar and dissimilar countries in order to better guide public health efforts to reduce dementia stigma.

\section{DISCLOSURE STATEMENT}

Authors' disclosures available online (https:// www.j-alz.com/manuscript-disclosures/21-0277r1).

\section{REFERENCES}

[1] World Health Organization (2017) WHO global action plan in the public health response to dementia 2017 - 2025. World Health Organization, Geneva.

[2] Herrmann LK, Welter E, Leverenz J, Lerner AJ, Udelson N, Kanetsky C, Sajatovic M (2018) A systematic review of dementia-related stigma research: Can we move the stigma dial? Am J Geriatr Psychiatry 26, 316-331.

[3] Nguyen T, Li X (2020) Understanding public-stigma and self-stigma in the context of dementia: A systematic review of the global literature. Dementia 19, 148-181.

[4] Werner P, Vermeulen P, Van Gorp B, Simonsen P (2021) From history to intervention: A socio-cultural analysis of dementia stigma. In Dementia and Society: An Interdisciplinary Approach, Vandenbulcke M, Dröes RM, Schokkaert E, ed. Cambridge University Press.

[5] Chow S, Chow R, Wan A, Lam HR, Taylor K, Bonin K, Rowbottom L, Lam H, DeAngelis C, Herrmann N (2018) National dementia strategies: What should Canada learn? Can Geriatr J 21, 173-209.

[6] Angermeyer MC, Matschinger H, Schomerus G (2013) Attitudes towards psychiatric treatment and people with mental illness: Changes over two decades. Br J Psychiatry 203, 146-151.
[7] Blay SL, Peluso ETP (2010) Public stigma: The community's tolerance of Alzheimer disease. Am J Geriatr Psychiatry 18, 163-171.

[8] Piver LC, Nubukpo P, Faure A, Dumoitier N, Couratier P, Clement P (2013) Describing perceived stigma against Alzheimer's disease in a general population in France: The STIG-MA survey. Int J Geriatr Psychiatry 28, 933-938.

[9] von dem Knesebeck O, Angermeyer MC, Ludecke D, Kofahl C (2014) Emotional reactions toward people with dementia - results of a population survey from Germany. Int Psychogeriatr 26, 435-441.

[10] Woo BK (2013) Knowledge of dementia among Chinese American immigrants. Asian J Psychiatr 6, 351-352.

[11] Johnson R, Harkins K, Cary M, Sankar P, Karlwish J (2015) The relative contributions of disease label and disease prognosis to Alzheimer's stigma: A vignette-based experiment. Soc Sci Med 143, 117-127.

[12] Stites SD, Rubright JD, Karlawish J (2018) What features of stigma do the public most commonly attribute to Alzheimer's disease dementia? Results of the survey of the U.S. general public. Alzheimers Dement 14, 925-932.

[13] Werner P, Kalaitzaki A, Spitzer N, Raviv L, Koukoli S, Tziraki Ch (2019) Stigmatic beliefs towards persons with dementia: Comparing Israeli and Greek college students. Int Psychogeriatr 31, 1393-1401.

[14] Adebiyi AO, Fagbola MA, Olakehinde O, Ogunniyi A (2016) Enacted and implied stigma for dementia in a community in south-west Nigeria. Psychogeriatrics 16, 268-273.

[15] Phillipson L, Magee C, Jones S, Skladzien E (2012) Exploring dementia and stigma beliefs: A pilot study of Australian adults aged 40 to 65 (Paper 28). Wollongong, NSW, Australia, University of Wollongong. https:// www.dementia.org.au/sites/default/files/Stigma_Report.pdf

[16] Umegaki H, Suzuki Y, Ohnishi J, Iguchi A (2009) Changes in the perception of dementia in Japan. Int Psychogeriatr 21, 793-795.

[17] Werner P, Davidson M (2004) Emotional reactions of lay persons to someone with Alzheimer's disease. Int J Geriatr Psychiatry 19, 391-397.

[18] Werner P (2008) Discriminatory behavior towards a person with Alzheimer's disease: Examining the effects of being in a nursing home. Aging Ment Health 12, 786-794.

[19] Cheng ST, Lam LCW, Chan LCK, Law ACB, Fung AWT, Chan WC, Tam CWC, Chan WM (2011) The effects of exposure to scenarios about dementia on stigma and attitudes toward dementia care in a Chinese community. Int Psychogeriatr 23, 1433-1441.

[20] Cheston R, Hancock J, White P (2016) A cross-sectional investigation of public attitudes toward dementia in Bristol and South Gloucestershire using the approaches to dementia questionnaire. Int Psychogeriatr 28, 1717-1724.

[21] Dao NCT, Woo BKP (2014) Gender differences in perceived stigma of dementia in Chinese Americans. Int $J$ Geriatr Psychiatry 29, 987-990.

[22] McParland P, Devine P, Innes A, Gayle V (2012) Dementia knowledge and attitudes of the general public in Northern Ireland: An analysis of national survey data. Int Psychogeriatr 24, 1600-1613.

[23] Phillipson L, Magee C, Jones S, Skladzien E (2014) Correlates of dementia attitudes in a sample of middle aged Australian adults. Australas J Ageing 33, 158-163.

[24] Abdullah T, Brown TL (2011) Mental illness stigma and ethnocultural beliefs, values, and norms: An integrative review. Clin Psychol Rev 31, 934-948. 
[25] Bosco A, Schneider J, Coleston-Shields DM, Higgs P (2019) The social construction of dementia: Systematics review and metacognitive model of enculturation. Maturitas 120, $12-22$.

[26] Justiss MD, Boustani M, Fox C, Katona C, Perkins AJ, Healey PJ, Sachs G, Hui S, Callahan CM, Hendrie HC, Scott E (2009) Patients' attitudes of dementia screening across the Atlantic. Int J Geriatr Psychiatry 24, 632-637.

[27] World Bank https://data.worldbank.org/indicator/SP.POP.65UP.TO.ZS

[28] OECD (2017) Dementia prevalence. In Health at a Glance 2017: OECD indicators. OECD Publishing, Paris.

[29] Australian Health Ministers Advisory Council (AHMAC) (2015) National Framework for action on Dementia 20152019.

[30] Brodsky J, Bentur N, Laron M, Ben-Israel S (2013) Addressing Alzheimer's and other types of dementia: Israeli National Strategy. http://www.health.gov.il/Publi cationsFiles/Dementia_strategy-Eng.pdf.

[31] Thomas DC (2008) Comparing cultures: Systematically describing cultural differences. In Cross-Cultural Management: Essential Concepts, Sage Publications, Thousand Oaks, CA, pp. 47-69.

[32] Papadopoulos C, Foster J, Caldwell K (2013) 'Individualism-collectivism' as an explanatory device for mental illness stigma. Community Ment Health J 49, 270-280.

[33] Aboseif A, Woo BKP (2020) The stigma of dementia. Genet Behav Diet Dement 2, 633-645.

[34] Cahill S, Pierce M, Werner P, Darley A, Bobersky A (2015) A systematic review of public's knowledge and understanding of Alzheimer's disease and dementia. Alzheimer Dis Assoc Disord 29, 255-275.

[35] Chang CY, Hsu HC (2020) Relationship between knowledge and types of attitudes towards people living with dementia. Int J Environ Res Public Health 17, 3777.

[36] Evans SC (2018) Ageism and dementia. In Contemporary Perspectives on Ageism, Ayalon L, Tesch-Römer C, eds. NY, Springer Open, pp. 263-276.

[37] Corrigan PW, Green A, Lundin R, Kubiak MA, Penn DL (2001) Familiarity with and social distance from people who have serious mental illness. Psychiatr Serv 52, 953-958.

[38] Corrigan PW, Nieweglowski K (2019) How does familiarity impact the stigma of mental illness? Clin Psychol Rev 70, 40-50.

[39] Werner P, Gur A, Porat A, Zubedat M, Shinan-Altman S (2020) Medical students' help-seeking recommendations for a person with Alzheimer's disease: Relationships with knowledge and stigmatic beliefs. Clin Psychol Rev 46, 294302.

[40] Werner P, Goldberg S, Mandel S, Korczyn AD (2013) Gender differences in lay persons' beliefs and knowledge about Alzheimer's disease (AD): A national representative study of Israeli adults. Arch Gerontol Geriatr 56, 400-404.

[41] Bodner E, Lazar A (2008) Ageism among Israeli students: Structure and demographic influences. Int Psychogeriatr 20, 1046-1058.
[42] Fraboni M, Saltstone R, Hughes S (1990) The Fraboni Scale of Ageism (FSA): An attempt at a more precise measure of ageism. Can J Aging 9, 56-66.

[43] Koschorke M, Evans-Lacko S, Sartorius N, Thornicroft G (2017) Stigma in different cultures. In The Stigma of Mental Illness-End of the Story, Gaebel W, Roessler W, Sartorius N, eds. Cham, Springer, pp. 67-82.

[44] Alzheimer's Disease International (2019) World Alzheimer Report 2019: Attitudes to dementia, a global survey. Alzheimers Dement 16, e038255.

[45] Werner P, Raviv-Turgeman L, Corrigan PW (2020) The influence of the age of dementia onset on college students' stigmatic attributions towards a person with dementia. $B M C$ Geriatr 20, 1-6.

[46] Barbarino P, Lynch Ch, Bliss A, Dabas L, Alzheimer's Disease International (2019) From plan to impact II: The urgent need for action. ADI, London.

[47] Bentur N, Sternberg SA (2019) Dementia care in Israel: Top down and bottom-up processes. Isr J Health Policy Res $\mathbf{8}$, 22.

[48] Department of Health (2015) National Framework for Action on Dementia 2015-2019. Commonwealth of Australia, Canberra. https://www.health.gov.au/resources/ publications/national-framework-for-action-on-dementia2015-2019

[49] Kemmelmeier M (2016) Cultural differences in survey responding: Issues and insights in the study of response biases. Int J Psychol 51, 439-444.

[50] Sharabi M, Polin B, Yanay-Ventura G (2019) The effect of social and economic transitions on the meaning of work. Empl Relat 41, 724-739.

[51] DeLuca JS, Hwang J, Stepinsli L, Yanos PT (2020) Understanding explanatory mechanisms for racial and ethnic differences in mental health stigma: The role of vertical individualism and right-wing authoritarianism. J Ment Health, doi: 10.1080/09638237.2020.1836556

[52] Yi J (2018) Revisiting individualism-collectivism. A crosscultural comparison among college students in four countries. J Intercult Commun 47, 1-13.

[53] Kim S, Mortby M (2017) Dementia-related stigma, cultural diversity, and help seeking intentions. Innov Aging 1, 693.

[54] Holzinger A, Floris F, Schomerus G, Carta MG, Angermeyer MC (2012) Gender differences in public beliefs and attitudes about mental disorder in western countries: A systematic review of population studies. Epidemiol Psychiatr Sci 21, 73-85.

[55] Bartlett R, Gjernes T, Lotherington AT, Obstfelder A (2018) Gender, citizenship and dementia care: A scoping review of studies to inform policy and future research. Health Soc Care Comm 26, 14-26.

[56] Manago B, Bernice BA, Olafsdottir S (2019) Icelandic inclusion, German hesitation and American fear: A crosscultural comparison of mental-health stigma and the media. Scand J Public Health 47, 90-98. 